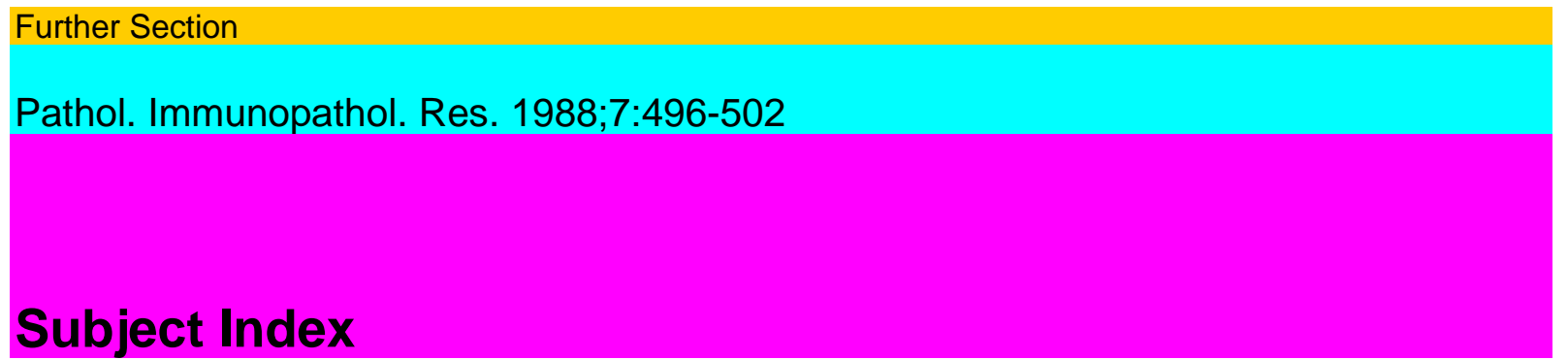

ABC-AP method 174,176,179

Acetylcholine receptor 321

Achondrodysplasia 59, 82, 83, 129

Achondrogenesis 90, 93

Achondroplasia 47, 86

Acquired immunodeficiency syndrome 345, 427,

483 Active myocarditis 229, 230 Acute heart failure, myocarditis 241 Acute lymphoblastic leukemia 327 Acute myeloblastic

leukemia 327 Acute myocarditis 246,247,251 Acute perimyocarditis 246 Acute respiratory distress syndrome 470 Adoptive immunotherapy 436, 442, 447 Adrenoleukodystrophy, X-linked 117 Alaskan malamute dog 63 Alizarin-prepared skeletons 140

Alkaline phosphatase 169,218

activity 172, 177 Alkaline phosphatase-antialkaline phosphatase

170

immunoalkaline phosphatase method 170, 174, 180 Alpha-interferon 467 Amines, lysosomotropic 158 Analysis, dual-color 310

Angiotensin II 200 Antibodies

antimyocardial 244, 245

antimyolemmal 245

antiplatelet 395, 402

antisarcolemmal 245

myelin-binding 165

virus-neutralizing 288 Antibody

anti-CVB3 259

antiplatelet 397

heart-specific 266, 276

virus-neutralizing 257

Antigen(s)

glial fibrillary acidic protein 210, 211

heart-specific 225, 274

proliferation-associated 372 Antithymocyte serum 297 Antitumor response, T-cell-specific 448 Aplastic anemia 324 Arachidonic acid metabolites 154 ARC

assay 201

brain 205 Arteriosclerosis 483 Autoantibodies 320

heart-specific 271 Autoimmune cytolytic T lymphocytes 282, 286 Autoimmune disorders 272, 319, 331, 426 Autoimmune hemolytic anemia 323 Autoimmune myocarditis 272, 274, 276, 285 Autoimmune response 271, 285 Autoimmune thrombocytopenia 323 Autoimmune thyroid disease 322 Autoimmunity 288, 296 cellular 285

picornavirus-induced 225, 279, 287 Autologous bone marrow transplantation 477, 478 Autolymphocyte therapy 483-486, 488, 490-492 Autolymphocytes 487, 489 Autotransplants 477 Avidin-biotin complex 170 Azathioprine 247, 298, 299

B cells 174, 305, 309-311, 315, 320, 326, 331, 357,

360-363, 365, 366, 411, 420, 421 B-cell neoplasms 331

Biochemical defects, chondrocyte-specific 56 Biopsy-proven myocarditis 298,301 Bone

dysplasias 76

marrow cells, immunofluorescence analysis 363

purging, autologous 477

Subject Index

497

Brachydactyly 109

Brachymorphic (bm/bm) mutant 56, 69 
Brachypodism (bp) 59

$\mathrm{Ca}^{2+}$ indicator dyes 419 Calcium

binding 414

channels 410, 426

concentration

cellular ionized 409-411 cytoplasmic 425

mobilization, fibronectin-induced 425

signalling 423

cytosolic 426 Calves, bulldog 64

Cancer, adoptive immunotherapy 433 Carboxypeptidase activity, angiotensin-related 200 Carcinogenesis, inhibition 187

Carcinogens 188, 191, 192 Cardiac allograft rejection 237 Cardiac myosin 274-276 Cardiomyopathy 225, 240, 252, 266

Cardiotropic virus 292 Cartilage 27, 146

collagens 20

growth plate 89

maldevelopment 107

matrix 27-29

deficiency 70,147

proteoglycans 28, 39, 86 Cattle, dwarfism 64 CB $_{3}$ infection 255, 267, 268, 272 CD designations 313 CD2 309, 423 CD3 309, 422 $\mathrm{CD}^{+}$cells 324 CD4 306,313,427

cells 283, 284, 322, 353, 419, 448 CD4/CD8 ratio 322-325 CD5 309 CD7 313 CD8 313,419

cells 312,322,323 CD8 ${ }^{+}$cells 324,353 CD 10 ${ }^{+}$cells 361,363,364 CD 16 antibodies 424 CD $16^{+}$cells 448 CD19 361 CD20

306,361,362 CDP, Conradi-Hunermann form 113

Cell

analysis, cytopreserved 353

surface antigens 358 Cells, bystander 149 CFgene 129 Chagas' disease 232, 235 Chemoprevention 183, 187, 195 Chondroblasts 33, 34 Chondrocalcin 30, 65 Chondrocyte phenotype 51 Chondrodysplasia(s) 26, 36, 55-57, 62, 63, 80-82,

87,99, 146

animal 55, 68

human 76, 78, 81, 85, 87, 99, 146

inherited 132

lethal neonatal 85

mouse 55, 57, 60

polygenic 62

punctata 110, 113, 115-117

single gene 62 Chondrodysplastic dwarfism 61 Chondrodystrophic mutant mice 58 Chondrodystrophies 48, 51 Chondrogenesis 24, 25, 32, 48, 59, 148

imperfecta 93 Choriocarcinoma 220 Chronic lymphocytic leukemia 319, 327

lymphocytes 329, 330 Ciclosporin 247, 296, 297, 301, 483 Cleft palate 78, 82 Cleidocranial dysplasia 139 Clinical flow cytometry, quality control 338 Coagulopathy, vitamin K-dependent 109 COL1A2 134 COL2A1 probe 105 Collagen(s) 14,20,27, 146

defect, type II 95

extraction 99

fibrillar 57

genes

cartilage 20 fibrillar 21

type(s) 91

type I 14, 16,34,39,87,91,93, 132-135

type II 5, 14, 16, 24-26, 36, 39, 48, 57, 71, 86, 87,90,91,93,95,96, 102, 104, 110

type III 15

type IX 21,22

typeX 14,15,17,18,22,48,65

type XII 21

498

Subject Index

Colon cancer 445

Common variable hypogammaglobulinemia 326

Congestive cardiomyopathy 235 
Conradi-Hunermann patients 114

Contour plots

light scatter 329

two-color 307,311,313 Corticosteroid therapy 297-299 Coxsackievirus B

infection 225, 235, 251, 260, 279

myocarditis, acute 245 Coxsackievirus B3 253-256, 258, 281, 288

infection 252, 282, 284, 288, 293 Coxsackieviruses 2, 254, 279 Cyclophosphamíde 297, 483 Cystic fibrosis 426 Cytokines 442, 447, 449, 484 Cytometer alignment 341 Cytotoxic T cells 269, 448, 471

Dallas criteria 243

Deficiency, cartilage matrix 56

De la Chapelle dysplasia 79

Diabetes, insulin-dependent 279, 288, 483

Dilated cardiomyopathy 240, 241, 244, 246, 247,

252, 279, 285 DMBA 192

DNA content measurements 371 Dot plots 308 Dot-blotting technique 181 DTH responses 438, 437 Duchenne's muscular dystrophy gene 120 Dwarfs 59, 60 Dyschondrodroplasia(s) 65

tibial 66 Dysgerminoma 209 Dysostosis multiplex 77 Dysplasia(s) 64, 65

family, diastrophic 77

bone 76,80

EB technique 392 EBV receptor 320 Echo viruses 240 Echocardiography 246 Ehlers-Danlos syndrome 123 Electrotransfer,

Western blot 181 ELISA 181

Embryonal carcinoma 208, 217, 219 EMC virus 293-295

Endocardial biopsy 234, 236, 242, 299, 302 Endodermal differentiation 213 Endomyocardial biopsy 227, 229, 231, 240, 245, 247

Endothelial cells

adherence of PMN 156

effects of IL-2 469 Endothelial damage 152-154,157 Endothelial leukocyte adhesion molecule 470 Endotoxins 232

Erythroid cells, light-scattering 359 Erythroleukemia 327 Extracellular matrix 34,35

f-MLP 425

FACS analyzer 389, 392, 393

Fibronectin 25, 30, 33, 34, 39, 40, 425

Flow cytometer 308, 353, 371, 398, 414, 417

Flow cytometric analysis 319, 339, 354, 372, 427

Flow cytometry 305, 306, 313, 316, 338, 353, 357,

381,421,427,484 Fluorescent reticulocyte count, TT 386 Fluorescent techniques 391 Focal myocarditis, virus-induced 276 Fura-2 $413,415,426$

\section{G-CSF 425}

Gamma-interferon 424, 446, 449, 450

Gating 306, 307, 342

Gene(s)

collagen 20

homeobox-containing 123, 124

hox 119

IL-1 472

procollagen 124

TNF 472

typeX 17

X-CGD 128 Germ cell tumors 219 Glial fibrillary acidic protein 208 Glucocorticoids 472 Glycophorin 406 GM-CSF 422, 425

Goldenhar syndrome 123 Graft-versus-host disease 477 Growth factors 43,49,451 Growth

hormone 191

plate $9,40,45$

cartilage 10, 13, 83 chondrocytes 47

Subject Index

499

Guillain-Barré syndrome 162 myelin antigen 166 
Hairy cell leukemia 175,178 Heart

disease, inflammatory 244,251

muscle disease, postmyocarditic 243, 244, 246 Hepatocellular carcinoma 219 HIV infection 345, 396 HLA class II antigens 174, 448 Homeobox 119, 120 Hox genes 120-124

Human myocarditis 225, 226, 240, 266, 297, 302 Human neoplasia 371 Huntington disease locus 129 Hypersensitivity myocarditis 235 Hypochondrodysplasia 83 Hypophosphatemic rickets 139

IAP level 491

IFN-gamma 435, 436

IL-1 449-451,467-470

IL-2 423, 433-439, 443, 444, 447, 449, 451, 452,

$459,460,463,468,469,483$

activated cells 450

adoptive immunotherapy 434, 439

cycle, gastrointestinal side-effects 465

immunotherapy 460, 472

infusions 460

plus LAK cell therapy 45, 444-446, 448, 450-453,459

receptor 284, 314, 434, 468, 469, 471, 491

soluble receptors, serum 491

stimulation 479

therapy 448, 462, 464-466

renal function abnormalities 462

toxicity 472

treatment 443, 447, 459, 469, 472 IL-2/LAK cell treatment 452, 467, 471 IL-2R 435,442,489,491 IL-4 468 IL-5 436

Immunity, virus-specific 282 Immunoalkaline phosphatase methods 169, 170,

173-178, 179-183 Immunoassay, enzyme-linked 399 Immunoblot 163, 165 Immunoblotting 96, 174, 181,245 Immunodeficiency, severe combined 326

Immunofluorescence

analysis 371

multicolor 358 Immunoglobulin, platelet-associated 395 Immunoperoxidase methods 173, 177, 181, 183 Immunophenotyping 319,356 Immunosuppressive therapy 292, 302 Immunotherapy 459, 483, 484 Incontinentia pigmenti 114 Indo-1 410-415, 417, 418, 422, 426, 427 Infectious mononucleosis 234 Infectious myocarditis 270 Infertility 483 Insulitis 280

Interferon 260, 294, 324, 483 Intergrin 35, 36

Intermediate filament proteins 371, 372 Ionomycin 417 Ischemic necrosis 226 ITP 398, 404, 407

Jurkat cells 420

K562 targets 470 Kneist

cartilage 96, 97

dysplasia 86,95,96, 123, 147

L3T4 antigen 284 LAK 434, 439, 443

activity 436, 437, 446, 449, 450, 453

cell

purging 477, 478, 480 therapy 447, 448, 459

cells 433, 434, 436, 439, 442, 448, 449, 451, 452, 459,460, 463, 477-480

effector cells $435,453,454,478$

phenomenon 433, 446, 447

therapy 45, 443, 444, 447, 450-452 Lambert-Eaton syndrome 426 Langer-Saldino

achondrogenesis 123,147

variant 90,93 Large granular lymphocytes 305, 423 Lasers, low power air-cooled 319 Leu-CAM complex 157 Leucogate 354

Leukemia, megakaryocytic 327 Leukotriene $\mathrm{B}_{4} 154$ Levamisole 182, 449

500

Subject Index

LGL/NK cells 307-312,316 Light scatter

forward 357, 364 
orthogonal 357, 364 Lipopolysaccharide 422 Lung cancer 445, 446 Lymphocyte

gating procedure 310,312

immunophenotyping 305, 331, 345, 354, 355

subset 350, 353, 354 Lymphocytic lymphoma 178 Lymphocytic vasculitis 438 Lymphokine-activated killer cells, see LAK cells Lymphokines 435, 442, 484 Lymphomas, non-Hodgkin’s 445 Lymphotoxin 450 Lysosomotropic agents 158

Mab, heart-reactive 287 Malignant hyperthermia 426 Malignant melanoma 438 Marfan syndrome 123,132 Matrix

Gla protein Ill

noncollagenous 27 McAlister dysplasia 79 Mean channel fluorescence 354 Melanoma 445, 446 Mice, (nu/nu) 267

Microfilaments 52, 53 Microglia 210,211 Migericin 417

Mixed lymphocyte culture 487 Monocytes, CD designation 316 Monocytes/macrophages 424, 442 Morquio syndrome 83 Mouse chondrodysplasias, monogenic 56

chondrodystrophic 60 Multiple sclerosis 321 Murine myocarditis 273, 296 Mutant

cartilage matrix deficiency 70

cmd/cmd 71 Myasthenia gravis 321 Myconcogene 33 Myelin proteins 16,162,163 Myeloid leukemia 327, 420 Myocardial

infarction 445 Myocardial pathology 296

Myocarditis 133, 225-227, 230-232, 234, 236, 243,

256, 258, 280, 288, 292, 293, 296, 299, 301, 302

acute 233, 235, 242, 245

biopsy-proven 299, 300

CB3-induced 258, 270, 296

chronic 234, 236, 237, 299

giant cell 229

histology 240, 243

human 294, 297

hypersensitivity 229

infectious 266, 269

murine models 267

pathology 228

postinfectious 266, 269, 271, 273, 275

viral 233, 235, 243, 247 Myositis 280

Nanomelic chondrocytes 73

Natural killer cells 256, 257, 259, 260, 269, 305, 325,

349, 355, 442, 447, 460, 470, 479 Neurofilament protein 208 Neuropeptides 451 Neutrophils 425 Nigerian 417 Nitrocellulose blots 163 Nonplatelet particles 406 Nonsteroidal anti-inflammatory drugs 297 Norwegian elkhound dog 63

Oligoclonal banding 321 Oncogene expression 32 Oncogenes 32, 33 Oncoproteins 372 Opsonins 152, 155, 156, 158 Ossification 110 Osteocalcin Ill

Osteochondrodysplasias 64, 140, 141 Osteochondrosis 65 Osteogenesis 51

imperfecta 82, 101, 132 Ovarian cancer 446

PAIg 396-400, 404, 405, 407 Parenti-Fraccaro variant 90 Perimyocarditis, acute 243 Peroxisomal diseases 113, 115-117 Phagocytes 149, 150, 154, 158 Phenotypic analysis 308, 313, 348 Photodetector 382 Picornavirus 240, 259, 279, 280 Subject Index

501

Pierre Robin sequence 104

Platelet antibody testing 400

Platelets 395-397, 399, 405, 412, 425

Platyspondyly 76

PMN 149-153, 157

PNS myelin proteins 162, 163, 165, 166

Prednisone 247, 301

Procollagen, type I 132

Prostaglandins, vasodilatory 462

Proteoglycan 52,68,71,74

Pseudoachondrodysplasia 87, 147 
QC procedures 338, 342 Quin2 415,417,427

Radioimmunoassay 202, 399 Receptor(s)

CVB3 288

p55 435

Sm-C/IGF-1 45,46 Refsum disease 117 Renal cell carcinoma 445, 486, 488, 490 Renin activity 201,205 Reticulocyte

concentration 387, 388

counts 381, 382, 384-386, 288, 392, 393

enumeration assay, cytometric 382 Retinoicacid 25

Rheumatoid arthritis 310, 319, 483 RNA dot blot 39 Ruddle hypothesis 123

Sarcoid heart disease 234

Sarcomas 446

Scatter contour plots, lymphocyte 330

SEDcongenita 100, 102

Selenium 187, 188, 190, 191, 193

Selenoproteins 194

Selenotrisulfides 193

SEMD 100, 102

Seminoma 209, 220

Short-limbed dwarfism, hydrops 90

Sickle cell anemia 128

Sjögren's syndrome 322

Smith-Lemli-Opitz 113

Solid tumors, multiparameter analysis 372

Somatomedin 40, 43, 47, 191

Southern blots 130

Spondyloepiphyseal dysplasia 92, 99

src oncogene 32, 36

Staphylococci 149, 151, 152, 156 Stickler syndrome 104-106 Streptococci, group A 275 Struma ovarii 216 Surface phagocytosis 152 Synaptosomal pellet 202, 204 Syndrome

Greig craniopoly sy ndactyly 123

Zellweger 115 Systemic lupus erythematosus 320, 483

Tcell(s) 259, 260, 267, 305, 309-315, 326, 327, 422,

423, 442, 447

CD4-positive 345,420

HIV-infected 41,417

receptor 272, 285, 288, 423

response, pathogenic 280

suppressor 269, $321 \mathrm{~T}$ lymphocytes 295, 345,413, 433, 439 Teratomas 205-211, 213-216, 320, 321 Thanatophoric dwarfism 92 Thanatophoric dysplasia 85 Thrombin 416,425 TIL 438

TNF 449, 450, 452, 467, 468, 470, 471 TNF-alpha (cachectin) 469 TNF-beta (lymphotoxin) 469 Toxicities, LAK plus IL-2 444 Toxicity, mechanism of IL-2 467 Toxins, endogenous 232 Toxoplasmosis 232 Trisomy 18, 113 TST 217

TT fluorescent technique 388, 391 Tumor

antigens, autologous 487

necrosis factor 435,467

regression 442 Tumor-infiltrating lymphocytes 434 Tumorigenesis 187-189 Tumors, germ cell 208 Type II

collagen 33, 34, 52, 56, 99, 101, 104, 129, 146

somatomedin receptor 45 Type IX genes 21 Type X collagen 14 Type XI collagen 135

Ulcerative colitis 483

502

Subject Index

Vasotocin 204

Viral antibody 269

Viral clearance 251, 254, 257 
Viral myocarditis 251,266,293,294

Virus

persistence 259, 260

receptor 286

vaccine 293 Virus-specific immunity 286

Warfarin embryopathy 107, 108, 110, Ill Western blot technique 182

X-linked agammaglobulinemia 326 X-linked mutant 55

Yolk sac tumor 208,217-219

Zellweger syndrome 115, 117 Zoo-blot 130 